\title{
ON THE STRUCTURE OF WEAKLY COMPACT SUBSETS OF HILBERT SPACES AND APPLICATIONS TO THE GEOMETRY OF BANACH SPACES
}

\author{
BY
}

\author{
S. ARGYROS AND V. FARMAKI
}

\begin{abstract}
A characterization of weakly compact subsets of a Hilbert space, when they are considered as subsets of $B$-spaces with an unconditional basis, is given. We apply this result to renorm a class of reflexive $B$-spaces by defining a norm uniformly convex in every direction. We also prove certain results related to the factorization of operators. Finally, we investigate the structure of weakly compact subsets of $L^{1}(\mu)$.
\end{abstract}

Introduction. A compact set is said to be uniformly Eberlein compact (U.E.C.) if it is homeomorphic to a weakly compact subset of a Hilbert space.

The class of U.E.C. sets has been introduced by Benyamini and Starbird in [4]. That paper contains an example of an Eberlein compact set (i.e. a weakly compact subset of $c_{0}(\Gamma)$ ) which is not U.E.C. Later Benyamini, Rudin and Wage [5] proved interesting stability properties for U.E.C. sets such as: The continuous Hausdorff image of a U.E.C. set is also U.E.C., and for any U.E.C. set $K$ the set $M_{1}^{+}(K)$ is also U.E.C. in the $w^{*}$-topology. In the same paper an internal topological characterization for U.E.C. sets was given. This result plays an important role in the proof of our results and its statement is given in the section of preliminaries (Proposition 0.3).

The present paper continues the study of U.E.C. sets. We prove a structure property for U.E.C. sets which are considered as subsets of $B$-spaces with an unconditional basis. This property, which also characterizes the U.E.C. sets, is, roughly speaking, a summability condition for the elements of the set. The statement of the result is as follows:

THEOREM A (1.8). Let $X$ be a B-space with an unconditional basis $\left\{x_{\gamma}: \gamma \in \Gamma\right\}$ and let $K$ be a weakly compact subset of $X$. Then the following are equivalent.

(1) The set $K$ is U.E.C.

(2) For every $\varepsilon>0$ there exists a decomposition $\left\{\Gamma_{m}^{(\varepsilon)}: m \in \mathbf{N}\right\}$ of $\Gamma$ and a sequence $\{k(m): m \in \mathbf{N}\}$ of natural members such that for any $x \in K$

$$
\operatorname{card}\left\{\gamma \in \Gamma_{m}^{(\varepsilon)}:\left|x_{\gamma}^{*}(x)\right| \geq \varepsilon\right\}<k(m)
$$

Received by the editors March 4, 1983 and, in revised form, June 1, 1984, September 11, 1984. 1980 Mathematics Subject Classification. Primary 46B20; Secondary 46B10, 46C10. 
The result is immediate when $K$ is considered as a weakly compact subset of a Hilbert space.

The proof of this theorem is given in the first section of the paper. We prove it for the special case $X=c_{0}(\Gamma)$ (Theorem 1.7). The proof strongly depends on the set $\mathscr{E}(K)$ introduced by Definition 1.1 and proceeds with the following two main steps. First, we show that, for any U.E.C. subset of $c_{0}(\Gamma)$, the set $\overline{\operatorname{co}}(\mathscr{E}(K))$ is also U.E.C. Second, we work with this set which has richer structure than the original $K$ and we obtain the desired result. The proof of the general case can be easily obtained by using the above result. Applying Theorem 1.7 we get a simplification of the example of Benyamini and Starbird [4] mentioned above (Example 1.10). This simplification also has been derived by an earlier unpublished work of the first named author in cooperation with S. Merkourakis.

In the second section we study the relation of U.E.C. sets with certain renormings of $B$-spaces. The main result proved here is Theorem 2.3 which states:

THEOREM B. Every reflexive B-space X, with its unit ball $S_{X}$ a U.E.C. set, admits an equivalent norm uniformly convex in every direction.

The definition of U.C.E.D. norm is given in the section of the preliminaries while certain results related to such norms, due to Troyanski, are given in the beginning of the second section.

The precise relation between $B$-spaces, containing only U.E.C. weakly compact subsets and possible renormings of them by an equivalent uniformly convex in every direction norm, is not yet clear. Besides Theorem B we investigate this relation by using an example (Example 2.7) and we state some related questions.

In the third section we introduce the class of uniformly weakly compact operators and we prove that any such operator can be factorized through a reflexive $B$-space admitting an equivalent uniformly convex in every direction norm. We also improve certain results of Davis, Figiel, Johnson and Pelczynski [6].

Finally, in the fourth section we show that every weakly compact subset of $L^{1}(\mu)$ is U.E.C.

Preliminaries. If $\Gamma$ is a set we denote its cardinality by $\operatorname{card}(\Gamma)$ or $|\Gamma|$. For a subset $A$ of $\Gamma$ we denote the indicator or characteristic function corresponding to the set $A$ by $I_{A}$. Below we state two combinatorial lemmas. They are both used in the proof of the main result of this paper.

0.1. Lemma [17]. Let $\left\{A_{i}: i \in I\right\}$ be an uncountable family of finite sets. Then there exists an uncountable subset $I^{\prime}$ of $I$ and a finite set $A$ such that for any two $i_{1} \neq i_{2}$ elements of $I^{\prime}$ we have $A_{i_{1}} \cap A_{i_{2}}=A$.

0.2. Lemma [3]. For any two natural numbers $k, \lambda$ and any family $\left\{A_{i}: i=1, \ldots, k^{\lambda}\right\}$ of sets with $\operatorname{card}\left(A_{i}\right) \leq \lambda$, there exists a subset $I$ of $\left\{1, \ldots, k^{\lambda}\right\}$ with card $I=k$ so that for $i_{1} \neq i_{2}$ elements of $I$ we have $i_{1} \notin A_{i_{2}}$.

The following proposition is an internal characterization of the U.E.C. sets and it is similar to the corresponding characterization for Eberlein compact sets proved by 
Rosenthal in [16]. Before the statement of the proposition we give some related definitions.

A family of sets $\left\{V_{\delta}: \delta \in \Delta\right\}$ separates the points of a given set $K$ if, for any $x, y \in K, x \neq y$, there exists $\delta \in \Delta$ so that $I_{V_{\delta}}(x) \neq I_{V_{\delta}}(y)$. The family $\left\{V_{\delta}: \delta \in \Delta\right\}$ is pointwise countable (finite) if every $x$ belongs to countably (finitely) many elements of the family. The family $\left\{V_{\delta}: \delta \in \Delta\right\}$ is $k$-finite for, a given natural number $k$, if any $x$ belongs to at most $k$ elements of the family.

0.3. Proposition [5]. A compact set $K$ is U.E.C. iff there exists a family $\left\{V_{\delta}\right.$ : $\delta \in \Delta\}$ of open $F_{\sigma}$ subsets of $K$ so that:

(i) The family $\left\{V_{\delta}: \delta \in \Delta\right\}$ separates the points of $K$.

(ii) There exists a decomposition of $\Delta$ into a sequence $\left\{\Delta_{n}\right\}_{n=1}^{\infty}$ and natural numbers $\{k(n)\}_{n=1}^{\infty}$ so that $\Delta_{n}$ is $k(n)$-finite.

For a set $\Gamma$ we denote by $c_{0}(\Gamma)$ the $B$-space of all functions $f$ on $\Gamma$ such that for all $\varepsilon>0$ the set $\{\gamma \in \Gamma:|f(\gamma)| \geq \varepsilon\}$ is finite. The norm on $c_{0}(\Gamma)$ is the sup norm. Also, $l^{2}(\Gamma)$ denotes the Hilbert space of all functions $f$ on $\Gamma$ such that $\|f\|=$ $\left(\sum_{\gamma \in \Gamma}|f(\gamma)|^{2}\right)^{1 / 2}<\infty$. In the sequel we denote the function $I_{\{\gamma\}}$ by $e_{\gamma}$. A family $\left\{x_{\gamma}: \gamma \in \Gamma\right\}$ in a $B$-space $X$ is an unconditional basis provided that it generates the space $X$ and, for any $\left\{x_{\gamma_{1}}, \ldots, x_{\gamma_{n}}\right\}$ finite subset of $\Gamma,\left\{a_{i}\right\}_{i=1}^{n}$ real numbers and $\left\{\varepsilon_{i}\right\}_{i=1}^{n} \in\{-1,1\}^{n}$

$$
\left\|\sum_{i=1}^{n} a_{i} x_{\gamma_{i}}\right\|=\left\|\sum_{i=1}^{n} \varepsilon_{i} a_{i} x_{\gamma_{i}}\right\| .
$$

Our notation and terminology for $B$-spaces and operators defined on them are those of [14].

0.4 . Definition. A $B$-space $X$ is said to be uniformly convex in every direction (U.C.E.D.) iff, for all sequences $\left\{x_{n}\right\}_{n=1}^{\infty},\left\{y_{n}\right\}_{n=1}^{\infty}$ such that $\left\|x_{n}\right\| \leq 1,\left\|y_{n}\right\| \leq 1$, $\left\|x_{n}+y_{n}\right\| \rightarrow 2$ and $x_{n}-y_{n}=\lambda_{n} z$, it follows that $\lambda_{n} \rightarrow 0$.

U.C.E.D. $B$-spaces were introduced by Garkavi [11]. Their structure has been studied by Day, James, and Swaminathan [7], Zizler [20], and Troyanski [18, 19]. Every separable $B$-space admits an equivalent U.C.E.D. norm [7], but Kutzarova and Troyanski in [13] gave an example of a reflexive $B$-space not admitting an equivalent U.C.E.D. norm. Also, any $B$-space which is U.C.E.D. satisfies the fixed point property [20].

1.1. Definition. In the sequel for any subset $K$ of $c_{0}(\Gamma)$ we will denote by $\mathscr{E}(K)$ the set

$$
\mathscr{E}(K)=\left\{x \in c_{0}(\Gamma): \exists y \in K, \text { such that }|x|=|y|\right\}
$$

and by $\mathscr{E}_{1}(K)$ the set

$$
\mathscr{E}_{1}(K)=\left\{x \in x_{0}(\Gamma): \exists y \in K, \text { such that }|x| \leq|y|\right\} .
$$

Here $|x|$ denotes the element of $c_{0}(\Gamma)$ with $|x|(\gamma)=|x(\gamma)|$ and $x \leq y$ denotes coordinatewise order.

Clearly, the definitions of $\mathscr{E}(K), \mathscr{E}_{1}(K)$ can be extended in the case of $B$-spaces with an unconditional basis. 
The following result is due to Abramovich [1].

1.2. Proposition. For any weakly compact subset $K$ of a $B$-space with an unconditional basis, the sets $\mathscr{E}(K), \mathscr{E}_{1}(K)$ are also weakly compact.

One of our purposes is to prove a corresponding result when $K$ is a U.E.C. subset of $c^{0}(\Gamma)$.

1.3. Proposition. Let $K$ be a weakly compact subset of $c_{0}(\Gamma)$. If $K$ is U.E.C., then the set $\mathscr{E}(K)$ is also U.E.C.

In order to prove it we use the following lemma.

1.4. Lemma. Consider $K$, a weakly compact subset of $l^{2}(\Lambda)$, and $\left\{V_{t}\right\}_{t \in T}, a$ pointwise countable family of open $F_{\sigma}$ subsets of $K$, so that $0 \notin V_{t}$ for all $t \in T$.

Then there exists a family $\left\{U_{\delta}\right\}_{\delta \in \Delta}$ of open $F_{\sigma}$ subsets of $K$ such that:

(1) For each $\delta$ in $\Delta$ there exists $t \in T$ so that the set $V_{t}$ contains the set $U_{\delta}$.

(2) For each $t$ in $T$ there exists a countable subset $\Delta^{t}$ of $\Delta$ such that

$$
V_{t}=\bigcup\left\{U_{\delta}: \delta \in \Delta^{t}\right\} .
$$

(3) $\Delta=\bigcup_{k=1}^{\infty} \Delta_{k}$, where for each $k \in \mathbf{N}$ the family $\left\{U_{\delta}\right\}_{\delta \in \Delta_{k}}$ is $n$ (k)-finite for some $n(k) \in \mathbf{N}$.

Proof. We assume $\|x\| \leq 1$ for all $x \in K$. For every $x$ in $V_{t}$ we choose a basic open neighborhood $W_{x}$ of $x$ contained in $V_{t}$. With no loss of generality we may assume that $W_{x}$ has the form

$$
W_{x}=\left\{y \in K:\left|y\left(\lambda_{i}^{x}\right)-x\left(\lambda_{i}^{x}\right)\right|<\varepsilon_{x}, i=1, \ldots, n_{x}\right\},
$$

where $n_{x} \in \mathbf{N}, \Lambda_{x}=\left\{\lambda_{i}^{x}\right\}_{i=1}^{n_{x}}$ is a finite subset of $\Lambda$, and $\varepsilon_{x}$ is a rational number. Furthermore, setting $M_{x}=\left\{\lambda_{i}^{x}:\left|x\left(\lambda_{i}^{x}\right)\right|>0\right\}$, we assume that $\varepsilon_{x}$ has been chosen so that $|x(\lambda)|>\varepsilon_{x} / 2$ for all $\lambda \in M_{x}$. We notice that because of the property $0 \notin W_{x}$ the set $M_{x}$ is nonempty.

Each $V_{t}$ is Lindelöf; hence, there exist countably many elements of it, say $\left\{x_{n}^{t}\right\}_{n=1}^{\infty}$, so that

$$
V_{t}=\bigcup_{n=1}^{\infty} W_{x_{n}^{\prime}} .
$$

Claim. For a fixed finite subset $M$ of $\Lambda$ and a fixed $k \in N$, the set

$$
L=\left\{t \in T: M_{x_{h}^{\prime}}=M\right\}
$$

is at most countable.

Proof of The Claim. Suppose on the contrary that there exist $k, M$ such that the corresponding set $L$ is uncountable. For each $t \in L$ we set $D_{t}=\Lambda_{x_{k}^{t}} \backslash M$. Applying Lemma 0.1 to the family $\left\{D_{t}\right\}_{t \in L}$, we get an uncountable subset $I$ of $L$ and a subset $D$ of $\Lambda$ with $D_{t_{1}} \cap D_{t_{2}}=D$ for any distinct elements $t_{1}, t_{2}$ of $I$. We also assume that, for any $t, t^{\prime} \in I, \varepsilon_{x_{k}^{\prime}}=\varepsilon_{x_{k}^{\prime}}=\varepsilon$, and that $\left|x_{k}^{t}(\lambda)-x_{k}^{t^{\prime}}(\lambda)\right|<\varepsilon / 2$ for each $\lambda \in M$. Fix $t_{0} \in I$ and consider the set

$$
\Gamma_{0}=\left\{\lambda \in \Lambda: x_{k}^{t_{0}}(\lambda) \neq 0\right\}
$$


which clearly is at most countable. Hence there is an uncountable $I_{1} \subset I$ with $D_{t} \cap \Gamma_{0} \subset D$ for any $t \in I_{1}$. We can easily see that the element $x_{k}^{t_{0}}$ belongs to the set $W_{x_{h}^{\prime}}$ for all $t \in I_{1}$. Hence there are uncountably many $V_{t}$ with nonempty intersection. This contradicts our assumptions and the proof of the Claim is complete.

Consequently, for each finite subset $M$ of $\Lambda$ and any $k \in \mathbf{N}$, the set

$$
\left\{W_{x_{k}^{\prime}}: M_{x_{k}^{\prime}}=M\right\}
$$

is at most countable. We enumerate this as

$$
\{W(M, r): r \in \mathbf{N}\} .
$$

Define $\Delta=T \times \mathrm{N}$ and $U_{\delta}=W_{x_{k}^{\prime}}$ where $\delta=(t, k)$. It is easy to see that the family $\left\{U_{\delta}: \delta \in \Delta\right\}$ satisfies the desired properties (1) and (2) of the statement of the lemma. It remains to show the last one. For this purpose, define

$$
\Delta(k, m, l, r)=\left\{\delta=(t, k): \varepsilon_{x_{k}^{t}}>1 / m,\left|M_{x_{k}^{\prime}}\right|=l, W_{x_{k}^{\prime}}=W(M, r)\right\} .
$$

Obviously,

$$
\Delta=\bigcup\left\{\Delta(k, m, l, r):(k, m, l, r) \in \mathbf{N}^{4}\right\} .
$$

For any $m, l \in \mathbf{N}$, we denote by $s(m, l)$ the cardinality of the set

$$
\left\{A \subset\left\{1, \ldots, m^{2}+1\right\}:|A|=l\right\} .
$$

We claim that the family $\left\{U_{\delta}: \delta \in \Delta(k, m, l, r)\right\}$ is $s(m, l)$-finite.

To see this we choose, whenever it exists, element $y$ of $K$ with

$$
y=\bigcap_{i=1}^{s(m, l)} U_{\delta_{i}} \text { for }\left\{\delta_{1}, \ldots, \delta_{s(m, l)}\right\} \subset \Delta(k, m, l, r) .
$$

Then there exists $\left\{\lambda_{1}, \ldots, \lambda_{m^{2}+1}\right\} \subset \bigcup_{i=1}^{s(m, l)} M_{x_{k_{i}^{\prime}}}$, where $\delta_{i}=\left(t_{i}, k_{i}\right)$. Also $\left|y\left(\lambda_{i}\right)\right|>$ $1 / m$ for all $i=1, \ldots, m^{2}+1$; hence, we get

$$
\|y\|=\left(\sum_{\lambda \in \Lambda}|y(\lambda)|^{2}\right)^{1 / 2} \geq\left(\sum_{i=1}^{m^{2}+1}\left|y\left(\lambda_{i}\right)\right|^{2}\right)^{1 / 2}>1 .
$$

This is a contradiction and the proof of the lemma is complete.

Before the proof of Proposition 1.3 we introduce some more notation for the sake of simplicity.

Notation. We fix an enumeration $\left\{\left(a_{n}, b_{n}\right): n \in \mathbf{N}\right\}$ of the open intervals with rational ends such that $0 \notin\left[a_{n}, b_{n}\right]$. Let $K$ be a subset of $c_{0}(\Gamma)$.

For any $(\gamma, n)$ in $\gamma \times \mathbf{N}$, we set

$$
V_{(\gamma, n)}=\left\{x \in K: x(\gamma) \in\left(a_{n}, b_{n}\right)\right\} .
$$

The family $\left\{V_{(\gamma, n)}:(\gamma, n) \in \Gamma \times \mathbf{N}\right\}$ satisfies the following properties.

(i) Each $V_{(\gamma, n)}$ is an open $F_{\sigma}$ subset of $K$ and does not contain the zero element.

(ii) It separates the points of $K$.

(iii) It is pointwise countable.

Proof of Proposition 1.3. We may assume that the set $K$ satisfies $x(\gamma) \geq 0$ for any $x \in K, \gamma \in \Gamma$. (Otherwise we consider the mapping $G: K \rightarrow K_{1}$, defined by 
$G(x)=|x|$, which is continuous, and by [5] the set $K_{1}=G[K]$ is U.E.C. It is clear that $\mathscr{E}(K)=\mathscr{E}\left(K_{1}\right)$.)

Consider a homeomorphism $f: K \rightarrow l^{2}(\Lambda)$ so that $\|f(x)\| \leq 1$ for any $x \in K$ and $f(0)=0$. In the sequel, $V_{(\gamma, n)}$ are subsets of $K$ as in the previous notation. The family $\left\{f\left[V_{(\gamma, n)}\right]:(\gamma, n) \in \Gamma \times \mathbf{N}\right\}$ is pointwise countable and separates the points of the set $f[K]$. Hence this family satisfies the assumptions of Lemma 1.4, so there exists another family $\left\{A_{\delta}\right\}_{\delta \in \Delta}$ of open $F_{\sigma}$ subsets of $f[K]$ which satisfies the conclusions of Lemma 1.4 .

Set $U_{\delta}=f^{-1}\left[A_{\delta}\right]$. It is clear that the families $\left\{V_{(\gamma, n)}:(\gamma, n) \in \Gamma \times \mathbf{N}\right\}$ and $\left\{U_{\delta}\right\}_{\delta \in \Delta}$ satisfy properties (1), (2) and (3) of the conclusion of Lemma 1.4.

For any $x \in U_{\delta}, \delta \in \Delta$, we choose an open neighborhood $V_{x}$ of $x$ contained in $U_{\delta}$ which, furthermore, has the form

$$
V_{x}=\left\{y \in K: y\left(\gamma_{i}^{x}\right) \in\left(c_{i}^{x}, d_{i}^{x}\right), i=1, \ldots, n_{x}\right\},
$$

where, for $1 \leq i \leq n_{x}$ and $\gamma_{i}^{x} \in \Gamma, c_{i}^{x}$ and $d_{i}^{x}$ are rational numbers such that they are both positive in the case $x\left(\gamma_{i}^{x}\right)>0$ and $c_{i}^{x}=-d_{i}^{x}$ otherwise.

Each $U_{\delta}$ is contained in some of the sets $V_{(\gamma, n)}$; therefore, for each $\delta \in \Delta$ and $x \in U_{\delta}$, there exists $(\gamma, n)$ so that $\gamma_{i}^{x}=\gamma$ for some $1 \leq i \leq n_{x}$, and $\left(c_{i}^{x}, d_{i}^{x}\right)$ is a subset of $\left(a_{n}, b_{n}\right)$.

For each $\delta \in \Delta$ the set $U_{\delta}$ is Lindelöf so there exists a countable subset $\{x(\delta, \mu)$ : $\mu \in \mathbf{N}\}$ of $U_{\delta}$ so that $U_{\delta}=\bigcup_{\mu \in \mathbf{N}} V_{x(\delta, \mu)}$.

For $x \in K$ and $s \in\{-1,1\}^{n_{\mathrm{v}}}$, we define

$$
V_{x}^{s}=\left\{\psi \in \mathscr{E}(K): \psi\left(\gamma_{i}^{x}\right) \in\left(s(i) c_{i}^{x}, s(i) d_{i}^{x}\right), i=1, \ldots, n_{x}\right\} .
$$

We set

$$
E=\left\{V_{x(\delta, \mu)}^{s}: \delta \in \Delta, \mu \in \mathbf{N}, s \in\{-1,1\}^{n_{v(\delta, \mu)}}\right\}
$$

and we claim that $E$ separates the points of $\mathscr{E}(K)$. Indeed, for $x_{1}, x_{2}$ in $\mathscr{E}(K)$ with $x_{1} \neq x_{2}$ we distinguish two cases.

Case $1 .\left|x_{1}\right| \neq\left|x_{2}\right|$.

Then there exists a set of the form $V_{(\gamma, n)}$ so that $I_{V_{(\gamma, n)}}\left(\left|x_{1}\right|\right) \neq I_{V_{(\gamma, n)}}\left(\left|x_{2}\right|\right)$. Hence, there is $x(\delta, \mu)$ so that $I_{V_{v(\delta, \mu)}}\left(\left|x_{1}\right|\right) \neq I_{V_{1(\delta, \mu)}}\left(\left|x_{2}\right|\right)$ and, choosing a suitable $s \in$ $\{-1,1\}^{n_{v(\delta, \mu)}}$, we conclude that

$$
I_{V_{i(\delta, \mu)}^{i}}\left(x_{1}\right) \neq I_{V_{i(\delta, \mu)}}\left(x_{2}\right) .
$$

Case 2. $\left|x_{1}\right|=\left|x_{2}\right|$.

In this case there exists $\gamma$ in $\Gamma$ with $0 \neq x_{1}(\gamma)=-x_{2}(\gamma)$. We may assume that $x_{1}(\gamma)>0$. Hence there is, for $n \in \mathbf{N}, x_{1}(\gamma) \in\left(a_{n}, b_{n}\right)$, equivalently $x_{1} \in V_{(\gamma, n)}$ while $x_{2} \notin V_{(\gamma, n)}$. It is immediate that there is $(\delta, \mu) \in \Delta \times \mathbf{N}$ such that $x_{1} \in V_{x(\delta, \mu)}$ and $x_{2} \notin V_{x(\delta, \mu)}$.

We define a decomposition of $E$ as follows:

For $(k, \mu, l) \in \mathbf{N}^{3}$ and $s \in\{-1,1\}^{l}$ we set

$$
E(k, \mu, l, s)=\left\{V_{x(\delta, \mu)}^{s}: \delta \in \Delta_{k}, s \in\{-1,1\}^{\prime}\right\} .
$$


Recall that the family $\left\{U_{\delta}: \delta \in \Delta_{k}\right\}$ is $n(k)$-finite. We claim that the family $E(k, \mu, l, s)$ is also $n(k)$-finite.

Indeed if there exists $y \in \bigcap_{i=1}^{n(k)} V_{x\left(\delta_{i}, \mu\right)}^{s}$ then there also exists an $x \in K$ with $|y|=x$ and $x \in \bigcap_{i=1}^{n(k)} V_{x\left(\delta_{1}, \mu\right)}$. The last implies that $\bigcap_{i=1}^{n(k)} U_{\delta_{i}} \neq \varnothing$. This contradiction establishes the Claim.

Using now the topological characterization of U.E.C. (Proposition 0.3) we get that the set $\mathscr{E}(K)$ is U.E.C. The proof of the proposition is complete.

1.5. Proposition. Let $K$ be a weakly compact subset of a B-space $X$ which is U.E.C. Then the set $K_{1}=\overline{\operatorname{co}}(K)$ is also U.E.C.

Proof. We denote, as usual, the set of all regular probability measures defined on the space $K$ endowed with the $w^{*}$ topology by $M_{1}^{+}(K)$. As it has been proved in [5] for $K$ a U.E.C. set, the set $M_{1}^{+}(K)$ is also U.E.C. Consider now the map $F$ : $M_{1}^{+}(K) \rightarrow \overline{\operatorname{co}}(K)$ which sends the measure $\mu$ to its barycenter. It is well known that $F$ is $w^{*}-w$ continuous and onto [15]. Therefore, $\overline{\operatorname{co}}(K)$ is U.E.C. since it is the continuous image of $M_{1}^{+}(K)$ [5].

1.6. LemMA. Let $K$ be a subset of $c_{0}(\Gamma)$ and let $L_{1}, L_{2}, \ldots, L_{n}$ be pairwise disjoint finite subsets of $\Gamma$. We assume that $p_{1} I_{L_{1}}+\cdots+p_{n} I_{L_{n}} \in \overline{\operatorname{co}}(\mathscr{E}(K))$. If a satisfies $0<a \leq \min \left\{p_{1}, \ldots, p_{n}\right\}$, then

$$
\text { a. }\left(I_{L_{1}}+\cdots+I_{L_{n}}\right) \in \overline{\operatorname{co}}(\mathscr{E}(K)) .
$$

Proof. First notice that, for $x \in \overline{\operatorname{co}}(\mathscr{E}(K))$ and a subset $A$ of $\Gamma$,

$$
\left.x\right|_{A}=\left(\left(\left.x\right|_{A}+\left.x\right|_{A} c\right)+\left(\left.x\right|_{A}-\left.x\right|_{A} c\right)\right) / 2 \in \overline{\operatorname{co}}(\mathscr{E}(K)) .
$$

Subsequently, suppose $x=p_{1} I_{L_{1}}+\cdots+p_{n+1} I_{L_{N+1}}$ belongs to $\overline{\operatorname{co}}(\mathscr{E}(K))$ and $a \in R$ satisfies $a \leq p_{1} \leq \cdots \leq p_{n+1}$. Observe that the element

$$
y=\frac{p_{1}}{p_{2}} x+\frac{p_{2}-p_{1}}{p_{2}}\left(p_{1} I_{L_{1}}\right)=p_{1} I_{L_{1} \cup L_{-2}}+\frac{p_{1}}{p_{2}}\left(p_{3} I_{L_{3}}+\cdots+P_{n+1} I_{L_{n+1}}\right)
$$

also belongs to $\overline{c o}(\mathscr{E}(K))$. Hence the lemma follows by induction.

1.7. TheOREM. Let $K$ be a weakly compact subset of $c_{0}(\Gamma)$. The following are equivalent.

(1) The set $K$ is U.E.C.

(2) For every $\varepsilon>0$ there exists a decomposition $\left\{\Gamma_{m}^{(\varepsilon)}: m \in \mathbf{N}\right\}$ of the set $\Gamma$ and $a$ sequence $\{k(m, \varepsilon): m \in \mathbf{N}\}$ of natural numbers so that for every $x$ in $K$ and $m \in \mathbf{N}$

$$
\operatorname{card}\left\{\gamma \in \Gamma_{m}^{(\varepsilon)}:|x(\gamma)|>\varepsilon\right\}<k(m, \varepsilon)
$$

Proof. We may assume that $\|x\| \leq 1$ for all $x \in K$.

(2) $\Rightarrow(1)$. For $n \in \mathbf{N}$ we set $A_{n}=\{j \in Z:-(n+1) \leq j \leq n+1, j \neq 0,-1\}$ and for $\gamma \in \Gamma$ and $j \in A_{n}$ we define

$$
V_{\gamma, j}^{n}=\{x \in K: j / n<x(\gamma)<(j+1) / n\} .
$$

It is easy to see that the family of open $F_{\sigma}$ sets

$$
\mathscr{H}=\left\{V_{\gamma, j}^{n}, \gamma \in \Gamma, n \in \mathbf{N}, j \in A_{n}\right\}
$$


separates the points of the set $K$. For $(n, m) \in \mathbf{N}^{2}$ we set

$$
\mathscr{H}_{(n, m)}=\left\{V_{\gamma, j}^{m}: \gamma \in \Gamma_{m}^{(1 / n)} \text { and } j \in A_{n}\right\} .
$$

Obviously, $\left\{\mathscr{H}_{(n, m)}\right\}_{(n, m) \in \mathbf{N}^{2}}$ defines a decomposition of the family $\mathscr{H}$ and each $\mathscr{H}_{(n, m)}$ is $\left(k(m, 1 / n) \cdot\right.$ card $\left.A_{n}\right)$-finite. Proposition 0.3 now implies that the set $K$ is U.E.C.

$(1) \Rightarrow(2)$. Consider the set $K_{1}=\overline{\operatorname{co}}\left(\mathscr{E}\left(K \cup\left\{e_{\gamma}: \gamma \in \Gamma\right\}\right)\right)$. Propositions 1.3 and 1.5 imply that the set $K_{1}$ is U.E.C. Hence there exists a homeomorphism $f$ : $K_{1} \rightarrow l^{2}(\Lambda)$ satisfying $f(0)=0$ and $\|f(x)\| \leq 1$ for every $x \in K_{1}$.

For a fixed number $\varepsilon, 0<\varepsilon<1$, and any $\gamma \in \Gamma$, we set $V_{\gamma}=\left\{x \in K_{1}:|x(\gamma)|>\right.$ $\varepsilon / 4\}$ and $d_{\gamma}=\varepsilon e_{\gamma} / 2$. Choose an open neighborhood $W_{\gamma}$ of $f\left(d_{\gamma}\right)$ contained in the set $f\left[V_{\gamma}\right]$ with the form

$$
W_{\gamma}=\left\{y \in f\left[K_{1}\right]:\left|f\left(d_{\gamma}\right)\left(\lambda_{i}^{\gamma}\right)-y\left(\lambda_{i}^{\gamma}\right)\right|<\varepsilon_{\gamma}, i=1, \ldots, n_{\gamma}\right\},
$$

where $\lambda_{1}^{\gamma}, \ldots, \lambda_{n_{\gamma}}^{\gamma} \in \Lambda$ and $\varepsilon_{\gamma}>0$. Notice that $0 \notin W_{\gamma}$. Hence, for at least one $i_{0} \in\left\{1, \ldots, n_{\gamma}\right\}, f\left(d_{\gamma}\right)\left(\lambda_{i_{0}}^{\gamma}\right) \neq 0$. Therefore we may choose $\varepsilon_{\gamma}$ so that, setting $\lambda_{i_{0}}^{\gamma}=\lambda_{\gamma}$, $0<\varepsilon_{\gamma}<\left|f\left(d_{\lambda}\right)\left(\lambda_{\gamma}\right)\right| / 2$.

So for any $y \in W_{\gamma}$ we have

$$
\left|y\left(\lambda_{\gamma}\right)\right|>\left|f\left(d_{\gamma}\right)\left(\lambda_{\gamma}\right)\right| / 2 .
$$

For each $\gamma \in \Gamma$ choose $U_{\gamma}$ an open basic neighborhood of $d_{\gamma}$ so that $U_{\gamma} \subset f^{-1}\left(W_{\gamma}\right)$ $\subset V_{\gamma}$ and with the form

$$
U_{\gamma}=\left\{x \in K_{1}:\left|x\left(\gamma_{j}\right)\right|<\varepsilon_{\gamma}^{1} \text { for } \gamma_{j} \in S_{\gamma} \backslash\{\gamma\}\left(\varepsilon-\varepsilon_{\gamma}^{1}\right) / 2<x(\gamma)<\left(\varepsilon+\varepsilon_{\gamma}^{1}\right) / 2\right\},
$$

where $S_{\gamma}$ is a finite subset of $\Gamma$ containing $\gamma$ and $0<\varepsilon_{\gamma}^{1}<\varepsilon / 2$.

Notice that the set $\left\{f\left(d_{\gamma}\right): \gamma \in \Gamma\right\}$ is a weakly discrete subset of $l^{2}(\Lambda)$ and $\left\{f\left(d_{\gamma}\right): \gamma \in \Gamma\right\} \cup\{0\}$ is weakly compact. Therefore, for any $\gamma \in \Gamma$ the set

$$
A_{\gamma}=\left\{\delta \in \Gamma: f\left(d_{\delta}\right)\left(\lambda_{\gamma}\right)>2 \varepsilon_{\gamma}\right\}
$$

is finite.

For $n, m$ natural numbers, set

$$
\Gamma_{(n, m)}^{(\varepsilon)}=\left\{\gamma \in \Gamma:\left|f\left(d_{\gamma}\right)\left(\lambda_{\gamma}\right)\right|>1 / n, \operatorname{card}\left(S_{\gamma} \cup A_{\gamma}\right)<m\right\} .
$$

It is obvious that

$$
\Gamma=\bigcup_{(n, m) \in \mathbf{N}^{2}} \Gamma_{(n, m)}^{(\varepsilon)}
$$

Claim. For any $x \in K_{1}$

$$
\operatorname{card}\left\{\gamma \in \Gamma_{n, m}^{(\varepsilon)}:|x(\gamma)|>\varepsilon\right\}<(2 n)^{2 m} .
$$

Proof of The Claim. Suppose on the contrary that the Claim fails for a pair $(n, m)$. This means that there exists $x \in K_{1}$ such that for the set $A=\left\{\gamma \in \Gamma_{(n, m)}^{(\varepsilon)}\right.$ : $|x(\gamma)|>\varepsilon\}$ we have card $A>(2 n)^{2 m}$. We apply Lemma 0.2 to the family $\left\{S_{\gamma} \cup A_{\gamma}\right.$ : $\gamma \in A\}$ and we get a subset $B$ of $A$ with card $B=(2 n)^{2}$ such that $\gamma_{1} \notin S_{\gamma_{2}} \cup A_{\gamma_{2}}$ for $\gamma_{1} \neq \gamma_{2} \in B$. 
We show that $\bigcap_{\gamma \in B} U_{\gamma} \neq \varnothing$. Indeed, let $x_{1}=|x|$. Clearly $x_{1} \in K_{1}$ and hence (Lemma 1.6) $\left.x_{1}\right|_{B} \in K_{1}$; from this we get that the element $y=\varepsilon I_{B} / 2 \in K_{1}$. It is easy to see that $y \in \bigcap_{\gamma \in B} U_{\gamma}$ and from this we have that $f(y) \in \bigcap_{\gamma \in B} W_{\gamma}$. Consequently, by $(*),\left|f(y)\left(\lambda_{\gamma}\right)\right|>1 / 2 n$ holds for all $\gamma \in B$. We also notice that for any $\gamma_{1} \neq \gamma_{2} \in B$ we have $\lambda_{\gamma_{1}} \neq \lambda_{\gamma_{2}}$. Hence

$$
1 \geq j|f(y)| \geq\left(\sum_{\gamma \in B}\left(f(y)\left(\lambda_{\gamma}\right)\right)^{2}\right)^{1 / 2}>\left(\operatorname{card} B \frac{1}{(2 n)^{2}}\right)^{1 / 2}=1 .
$$

This contradiction proves the Claim and the proof of the theorem is complete.

The following theorem extends Theorem 1.7.

1.8. TheOREM. Let $X$ be a B-space with an unconditional basis $\left\{x_{\gamma}: \gamma \in \Gamma\right\}$ and let $K$ be a weakly compact subset of $X$. The following are equivalent.

(1) The set $K$ is U.E.C.

(2) For every $\varepsilon>0$ there exists a decomposition $\left\{\Gamma_{m}^{(\varepsilon)}: m \in \mathbf{N}\right\}$ of $\Gamma$ and a sequence $\{k(m): m \in \mathbf{N}\}$ of natural numbers such that for any $x \in K$

$$
\operatorname{card}\left\{\gamma \in \Gamma_{m}^{(\varepsilon)}:\left|x_{\gamma}^{*}(x)\right| \geq \varepsilon\right\}<k(m)
$$

Proof. We define a bounded linear one-to-one operator $T: X \rightarrow c_{0}(\Gamma)$ with the rule $T x(\gamma)=x_{\gamma}^{*}(x)$. Applying Theorem 1.7 to the set $T[K]$ which is homeomorphic to the set $K$ we get the desired result.

Restricting our interest to the class of U.E.C. sets containing only indicator functions, Theorem 1.8 can be formulated as

1.9. Corollary. Consider a weakly compact subset $K$ of a B-space $X$ with an unconditional basis $\left\{x_{\gamma}: \gamma \in \Gamma\right\}$ that has the form $\left\{I_{s}: S \in A\right\}$, for a family $\mathscr{D}$ of subsets of the set $\Gamma$. The set $K$ is a U.E.C. set iff there exists a decomposition $\left\{\Gamma_{m}\right.$ : $m \in \mathbf{N}\}$ of $\Gamma$ and a sequence $\{k(m): m \in \mathbf{N}\}$ of natural numbers satisfying

$$
\operatorname{card}\left(S \cap \Gamma_{m}\right)<k(m) \quad(S \in \mathscr{D}) \text {. }
$$

Applying Corollary 1.9 we simplify the example in [4] of a set $K$ which is Eberlein compact but not U.E.C.

1.10. Example. We denote by $\Gamma$ the set $\prod_{n=1}^{\infty}\{1, \ldots, n\}$. A subset $A$ of $\Gamma$ is said to be admissible if it satisfies the following condition.

There exists a natural number $n=n(A)$ so that for any $x$, $y \in A$ with $x \neq y$ we have $x(j)=y(j)$ for $j<n-1$ and $x(n) \neq y(n)$.

Consider the set $K=\left\{I_{A}: A \subset \Gamma, A\right.$ is admissible $\}$. We show that $K$ is a weakly compact subset of $c_{0}(\Gamma)$. To see this, we first notice that if $A$ is not admissible, there exist $\gamma_{1}, \gamma_{2}, \gamma_{3}$ elements of $A$ so that the set $\left\{\gamma_{1}, \gamma_{2}, \gamma_{3}\right\}$ also is not admissible. Therefore the set $K$ is a closed subset of the space $\{0,1\}^{\Gamma}$ endowed with the product topology, and since the weak topology of $c_{0}(\Gamma)$ coincides with the pointwise convergent topology we get that $K$ is indeed weakly compact. 
It remains to show that $K$ is not U.E.C. Assume that $K$ is U.E.C. From Corollary 1.9 there exists a decomposition $\left\{\Gamma_{m}: m \in \mathbf{N}\right\}$ of $\Gamma$ and natural numbers $\{k(m)\}_{n=1}^{\infty}$ so that card $\left(A \cap \Gamma_{m}\right)<k(m)$ for any admissible set $A$ and $m \in \mathbf{N}$. On the other hand, by Baire's theorem there exists an $m_{0} \in \mathbf{N}$ and a basic open subset $V=$ $\left(n_{1}, \ldots, n_{k}\right) \times \Pi_{n=k+1}^{\infty}\{1, \ldots, n\}$ of $\Gamma$ so that $\Gamma_{m_{0}} \cap V$ is dense in $V$. Hence we easily see that the set $\Gamma_{m_{0}}$ contains arbitrarily large admissible sets. This is a contradiction and the proof is complete.

2. We are now going to apply Theorems 1.7 and 1.8 in order to get equivalent U.C.E.D. norms on $B$-spaces. The results of this section also depend on two lemmas of Troyanski [18]. For the sake of completeness we state them below.

2.1. LemmA [18]. If a B-space $X$ admits a bounded linear one-to-one operator $T$ : $X \rightarrow l^{x}(\Gamma)$ so that:

For any $\varepsilon>0$ there exists a decomposition $\left\{\Gamma_{m}^{(\varepsilon)}: m \in \mathbf{N}\right\}$ of $\Gamma$ with the property:

$$
\text { For any } x \in X, \operatorname{card}\left\{\gamma \in \Gamma_{m}^{(\varepsilon)}:\|T x(\gamma)\|>\varepsilon\|T\|\|x\|\right\}<m \text {. }
$$

Then $X$ admits an equivalent U.C.E.D. norm.

2.2. Lemma [18]. A B-space with an unconditional basis $\left\{x_{\gamma}: \gamma \in \Gamma\right\}$ admits an equivalent U.C.E.D. norm iff:

For any $\varepsilon>0$ there exists a decomposition

$$
\left\{\Gamma_{m}^{(\varepsilon)}: m \in \mathbf{N}\right\} \text { of } \Gamma \text { such that }\left\|\sum_{k=1}^{m} x_{\gamma_{k}}\right\|>\frac{1}{\varepsilon}
$$

for every $m \in \mathbf{N}$ and subset $\left\{\gamma_{1}, \ldots, \gamma_{m}\right\}$ of $\Gamma_{m}^{(\varepsilon)}$.

2.3. Theorem. Any reflexive B-space $X$ whose unit ball is a U.E.C. set admits an equivalent U.C.E.D. norm.

Proof. By the fundamental theorem of Amir and Lindenstrauss [2] there exists a bounded one-to-one operator $T: X \rightarrow c_{0}(\Gamma)$. We assume that $\|T\|=1$ and hence the set $T\left[S_{X}\right]$ is a U.E.C. subset of the unit ball of $c_{0}(\Gamma)$. A combination of Theorem 1.7 and Lemma 2.1 gives the desired result.

As we mentioned in the section of preliminaries, every $B$-space $X$ with a U.C.E.D. norm satisfies the fixed point property. Hence Theorem 2.3 implies the following corollary.

2.4. Corollary. Every reflexive B-space whose unit ball is a U.E.C. set admits an equivalent norm satisfying the fixed point property.

The question whether the converse of Theorem 2.3 holds is still open. A partially positive answer to this problem is given by the following result.

2.5. Theorem. Let $X$ be a $B$-space with an unconditional basis. If $X$ admits an equivalent U.C.E.D. norm, then every weakly compact subset of $X$ is U.E.C.

Proof. Consider $K$ weakly compact subset of $X$. The set $K$ is bounded; therefore, there exists $\vartheta>0$ with $\|x\|<\vartheta$ for every $x \in K$. For fixed $\varepsilon>0$ set $\varepsilon^{\prime}=\varepsilon / \vartheta$ and 
define a decomposition $\left\{\Gamma_{m}^{\left(\varepsilon^{\prime}\right)}: m \in \mathbf{N}\right\}$ of $\Gamma$ so that the conclusion of Lemma 2.2 is satisfied.

We claim that for any $x \in K$

$$
\operatorname{card}\left\{\gamma \in \Gamma_{m}^{\left(\varepsilon^{\prime}\right)}:\left|x_{\gamma}^{*}(x)\right|>\varepsilon\right\}<m .
$$

Assume that this is not the case. Then there are $x_{0} \in K$ and $m_{0} \in \mathbf{N}$ so that for the set $A=\left\{\gamma \in \Gamma_{m_{0}}^{\left(\varepsilon^{\prime}\right)}:\left|x_{\gamma}^{*}\left(x_{0}\right)\right|>\varepsilon\right\}$ we have card $A \geq m_{0}$. Since $\left\{x_{\gamma}\right\}_{\gamma \in \Gamma}$ is an unconditional basis we get

$$
\left\|x_{0}\right\|>\varepsilon\left\|\sum_{\gamma \in A} x_{\gamma}\right\| \geq \varepsilon \frac{\vartheta}{\varepsilon}=\vartheta .
$$

This is a contradiction proving the Claim and the theorem.

In [13] an example is given of a reflexive $B$-space with an unconditional basis and without an equivalent U.C.E.D. norm. By the following result we show the existence of such a $B$-space using factorization techniques from [6].

2.6. Corollary. There exists a reflexive B-space with an unconditional basis and without an equivalent U.C.E.D. norm.

Proof. In [6] it is proved that every weakly compact set $W$ is topologically homeomorphic to a weakly compact subset of a reflexive $B$-space $X(W)$ with an unconditional basis (see 3.4 below). Let $W$ be the Benyamini-Starbird set (Example 1.10). Theorem 2.5 implies that $X(W)$ does not admit an equivalent U.C.E.D. norm, completing the proof.

The converse of Theorem 2.5 fails in general. We next give an example of a $B$-space $X$ with an unconditional basis so that every weakly compact subset of it is U.E.C. but no equivalent U.C.E.D. norm is defined on $X$.

2.7. Example. Consider the set $\Gamma=\prod_{n=1}^{\infty}\{1, \ldots, n\}$ and define the family $\mathscr{D}$ of admissible sets in the same fashion as in Example 1.10. Namely, an $A \subset \Gamma$ is admissible iff there exists $n \in \mathbf{N}$ such that for any $\gamma_{1}, \gamma_{2} \in A, \gamma_{1} \neq \gamma_{2}$, we have

$$
\gamma_{1}(j)=\gamma_{2}(j) \text { for } j<n \text { and } \gamma_{1}(n) \neq \gamma_{2}(n) \text {. }
$$

Define the $B$-space $X$ as the completion of the space of all real valued functions $\varphi$ from $\Gamma$ with finite support and with norm given by

$$
\|\varphi\|=\sup _{B \in \mathscr{D}}\left(\sum_{\gamma \in B}|\varphi(\gamma)|\right) .
$$

Set $e_{\gamma}=I_{\{\gamma\}}$ and notice that the family $\left\{e_{\gamma}: \gamma \in \Gamma\right\}$ is an unconditional basis for the space $X$. Denote by $W$ the $B$-space generated by the family $\left\{e_{\gamma}^{*}: \gamma \in \Gamma\right\}$ which, of course, is an unconditional basis for the space $W$.

For any admissible subset $B$ of $\Gamma$ it is easy to see that

$$
\left\|\sum_{\gamma \in B} e_{\gamma}\right\|=|B| \text { and }\left\|\sum_{\gamma \in B} e_{\gamma}^{*}\right\|=1
$$


FACT. Every weakly compact subset of $W$ is metrizable and hence U.E.C.

In order to prove it we need the following two lemmas.

Lemma A. For each infinite subset $\Delta$ of $\Gamma$ there is an infinite sequence $\left(\gamma_{j}\right) \subset \Delta$ so that $\left(e_{\gamma_{1}}^{*}\right)_{j}$ is 2-equivalent to the unit vectors of $l^{1}(\mathbf{N})$.

Proof. Assume $\Delta$ is countable. We decompose the set $\Delta^{[3]}$ of all three-point subsets of $\Delta$ into two sets $G, H$ as follows: We set $\left\{\gamma_{1}, \gamma_{2}, \gamma_{3}\right\}$ in $G$ iff it is admissible. By the well-known Ramsey theorem there exists an infinite subset $E$ of $\Delta$ so that either $E^{[3]} \subset G$ or $E^{[3]} \subset H$. The case $E^{[3]} \subset G$ implies that $E$ is admissible which is impossible. Hence the case $E^{[3]} \subset H$ remains which, equivalently, means that any admissible subset of $E$ contains at most two elements.

Consider the closed linear span $X_{E}$ of the family $\left\{e_{\gamma}: \gamma \in E\right\}$. We show that $X_{E}$ is 2-equivalent to the space $\mathrm{c}_{0}(\mathbf{N})$. Indeed, for any element $\varphi$ of $X_{E}$ with finite support we have

$$
\|\varphi\|=\sup _{\gamma_{1}, \gamma_{2} \in E:}\left\{\left|\varphi\left(\gamma_{1}\right)\right|+\varphi\left(\gamma_{2}\right) \mid\right\} \leq 2 \sup _{\gamma \in E:}|\varphi(\gamma)| \leq 2\|\varphi\| .
$$

The family $\left\{e_{\gamma}: \gamma \in \Gamma\right\}$ is an unconditional basis for the space $X$. Hence the family $\left\{e_{\gamma}^{*}: \gamma \in E\right\}$ is 2-equivalent to the unit vectors of $l^{1}(\mathbf{N})$ and the proof of the lemma is complete.

Lemma B. Let $K$ be a weakly compact subset of a B-space $X$ with an unconditional basis $\left\{e_{\gamma}: \gamma \in \Gamma\right\}$. Then the set $A=\left\{e_{\gamma}: \exists x \in K x(\gamma) \neq 0\right\} \cup\{0\}$ is o-weakly compact.

Proof. The set $W=\overline{\operatorname{co}}\left(\mathscr{E}_{1}(K)\right)$ is weakly compact [1]. Set $A_{n}=\left\{e_{\gamma}: \exists x \in K\right.$ $|x(\gamma)| \geq 1 / n\}$ and notice that $\left(A_{n} / n\right) \cup\{0\}$ is a subset of $W$. Hence $A_{n} \cup\{0\}$ is weakly compact provided that $A_{n} / n \cup\{0\}$ is a weakly closed subset of $W$. To see this choose a sequence $\left(e_{\gamma_{k}}\right)$ from $A_{n}$. The only possible limit point for weakly convergent subsequences of $\left(e_{\gamma_{k}} / n\right)_{k=1}^{\infty}$ is the element $0 \in X$. Therefore the set $\left(A_{n} / n\right) \cup\{0\}$ is sequentially closed and by Eberlein's criteria [9] it is weakly closed.

Proof of THE Fact. Consider a weakly compact subset $K$ of $W$. We show that the set

$$
D=\left\{e_{\gamma}^{*}: \exists x \in K x(\gamma) \neq 0\right\}
$$

is at most countable.

Indeed assuming that $D$ is uncountable, Lemma $\mathrm{B}$ implies that there exists a sequence $\left(e_{\gamma_{n}}\right)$ from $D$ weakly convergent to zero. On the other hand, by Lemma $\mathrm{A}$ we have that a subsequence $\left(e_{\gamma_{n_{k}}}\right)$ is equivalent to the unit vectors of $l^{1}(\mathbf{N})$. This is a contradiction proving that $D$ is countable and therefore $K$ is U.C.E.D. as a subset of a separable subspace of $W$.

We finally show that $W$ does not admit an equivalent U.C.E.D. norm. If it did, then by Lemma 2.2 for $\varepsilon=\frac{1}{2}$, there exists a decomposition $\left\{\Gamma_{m}^{(\varepsilon)}: m \in \mathbf{N}\right\}$ so that for each $m \in \mathbf{N},\left\{\gamma_{1}, \ldots, \gamma_{m}\right\} \subset \Gamma_{m}^{(\varepsilon)}$ we have

$$
\left\|\sum_{i=1}^{m} e_{\gamma_{t}}^{*}\right\|>2 .
$$


On the other hand, using Baire's theorem we find $m_{0} \in \mathbf{N}$ with $\Gamma_{m_{0}}^{(\varepsilon)}$ containing arbitrarily large admissible subsets. This is a contradiction since we have noted that for any admissible set $B$ we have $\left\|\sum_{\gamma \in B} e_{\gamma}^{*}\right\|=1$. This completes the procf of the desired properties of the example.

2.8. Remark. (a) We still do not know if there exists a W.C.G. $B$-space $X$ containing only U.E.C. weakly compact subsets and not admitting an equivalent U.C.E.D. norm. Candidates for counterexamples are the nonseparable $L^{1}(\mu)$-spaces, for $\mu$ finite measures. Indeed, the first author mentioned in the fourth section shows that the weakly compact subsets of these spaces are U.E.C. The existence of an equivalent U.C.E.D. norm on $L^{1}(\mu)$ does not seem known yet.

(b) The results of this section indicate a relation between the existence of a U.C.E.D. norm on a $B$-space $X$, which is a geometric property, and of the analytic property that each weakly compact subset of $X$ is U.E.C. The exact relation between them is not clear to us. In the case of reflexive $B$-spaces with an unconditional basis, Theorems 2.3 and 2.5 show the equivalence of these properties.

We state below the main open questions.

2.9. Problem. Let $X$ be a $B$-space with a U.C.E.D. norm.

(i) Assuming that $X$ is reflexive, is its unit ball U.E.C.?

(ii) In general, does $X$ contain only U.E.C. weakly compact subsets?

(c) The relation between U.E.C. sets and the fixed point property seems also interesting. Related to this is the following problem.

2.10. Problem. Let $X$ be a $B$-space containing only U.E.C. weakly compact subsets. Does there exist an equivalent norm on it satisfying the fixed point property?

3. In this section a new class of operators is introduced. We will call them uniformly weakly compact and we investigate their factorization through "nice" $B$-spaces.

3.1. Definition. A linear operator $T: X \rightarrow Y$ is called uniformly weakly compact if the weak closure of the set $T\left[S_{X}\right]$ is a U.E.C. subset of the space $Y$.

3.2. REMARK. Every U.W.C. operator is weakly compact. In general, the converse is not correct. The factorization theorem (see Notation 3.3 below) implies that every weakly compact subset of a $B$-space $Y$ is U.E.C. iff any weakly compact operator into $Y$ is U.W.C.

Our results are closely related to the factoring reflexive $B$-space $Y$ described in [6]. In the following notation we restate the main steps of the construction of this space as well as its basic properties.

3.3. Notation. Fix a convex symmetric weakly compact subset $W$ of a $B$-space $(X,\|\cdot\|)$.

For each $n=1,2, \ldots$ we denote by $\|\cdot\|_{n}$ the gauge of the set

$$
V_{n}=2^{n} W+2^{-n} S_{X}
$$

We define $\|x\| \|=\left(\sum_{n=1}^{\infty}\|x\|_{n}^{2}\right)^{1 / 2}$ and we set $Y=\{x \in X:\|x\|<\infty\}$. The following properties are proved in [6]. 
(1) $W \subseteq S_{Y}$.

(2) $(Y, \||\cdot| \mid)$ is reflexive.

(3) The identity $j: Y \rightarrow X$ is continuous.

(4) Assume that $X$ has an unconditional basis $\left\{x_{\gamma}: \gamma \in \Gamma\right\}$ and for any finite $F \subset \Gamma$ we have $P_{F}(W) \subset \lambda_{F} W$. (Here, $P_{F}$ denotes the usual projection of $X$ on to the space generated by the set $\left\{x_{\gamma}: \gamma \in F\right\}$.) Then the set $\left\{x_{\gamma}: \gamma \in \Gamma\right\} \cap Y$ is an unconditional basis for the space $Y$.

In the sequel, for given $X$ and $W$, the letters and symbols $Y, S_{Y}, j, V_{n},\|\cdot\|_{n}$ will be used in their above defined context.

3.4. Remark. Consider a weakly compact subset $K$ of a $B$-space $X$ with an unconditional basis $\left\{x_{\gamma}: \gamma \in \Gamma\right\}$. Abramovich [1] proved that the set $\mathscr{E}_{1}(K)$ (see Definition 1.1) is also weakly compact. Hence the set $W=\mathscr{E}_{1}(\overline{\operatorname{co}}(K \cup(-K)))$ satisfies the assumptions of condition (4) in Notation 3.3 and, consequently, we conclude that any Eberlein compact set $K$ is homeomorphically embedded into a reflexive $B$-space $Y$ with an unconditional basis [10].

3.5. LeMma. Consider a convex symmetric weakly compact subset $W$ of a B-space $X$ which is U.E.C. Then the unit ball $S_{Y}$ of the factoring space $Y$ is also U.E.C.

Proof. The identity map $j:(Y,\|\mid \cdot\|) \rightarrow(X,\|\cdot\|)$ is continuous. Therefore the $B$-space $Z$, the norm closure of $j[Y]$, is weakly compactly generated (W.C.G.) and by [2] there is a linear one-to-one $T: Z \rightarrow c_{0}(\Gamma)$ with $\|T\|=1$.

We wish to show that the weakly compact set $T \circ j\left[S_{Y}\right]$ satisfies condition (2) of Theorem 1.7. By our assumptions the set $W$ is a U.E.C. subset of $Z$; hence, $T[W]$ satisfies condition (2) of Theorem 1.7. Set $\varepsilon=1 / 2^{4 n^{2}}$ and we choose a decomposition $\left\{\Gamma_{m}^{(\varepsilon)}: m \in \mathbf{N}\right\}$ so that for any $x \in W$ and $m \in \mathbf{N}$,

$$
\operatorname{card}\left\{\gamma \in \Gamma_{m}^{(\varepsilon)}:|T(x)(\gamma)|>1 / 2^{4 n^{2}}\right\}<k(m) .
$$

Claim. For any $x \in S_{Y}$ and $m \in \mathbf{N}$,

$$
\operatorname{card}\left\{\gamma \in \Gamma_{m}^{(\varepsilon)}:|T \circ j(x)(\gamma)|>1 / n\right\}<k(m) .
$$

Proof of The Claim. Assume, on the contrary, that the relation $(* *)$ fails for some $x_{0} \in S_{Y}$ and $m_{0} \in \mathbf{N}$.

For fixed $k \in \mathbf{N}$ choose $\lambda>0$ such that $x_{0} \in \lambda V_{k}$ or, equivalently, $x_{0}=\lambda 2^{k} w+$ $2^{-k} \lambda y$ for some $w \in W$ and $y \in S_{X}$.

Notice that the last equality implies that $y$ is an element of the space $Z$.

By the relation (*) and the choice of $x_{0}$ we get a $\gamma_{0} \in \Gamma_{m_{0}}$ such that

$$
\left|T \circ j\left(x_{0}\right)\left(\gamma_{0}\right)\right|>1 / n \text { and }\left|T(w)\left(\gamma_{0}\right)\right|<1 / 2^{4 n^{2}} \text {. }
$$

By the linearity of $T$ we also have for all $\gamma \in \Gamma$

$$
T \circ j\left(x_{0}\right)(\gamma)=\lambda 2^{k} T(w)(\gamma)+\lambda 2^{-k} T(y)(\gamma) .
$$

Therefore for any $k \in \mathbf{N}$ and $\lambda \in \mathbf{R}$ such that $x \in \lambda V_{k}$ we have that

$$
1 / n \leq \lambda\left(2^{k} / 2^{4 n^{2}}+1 / 2^{k}\right) \leq \lambda\left(2^{k} / 2^{4 n^{2}}+1\right) .
$$

Recall that $\|x\|_{k}=\inf \left\{\lambda: x \in \lambda V_{k}\right\}$. Hence

$$
1 / n \leq\left\|x_{0}\right\|_{k}\left(2^{k} / 2^{4 n^{2}}+1\right) \text {. }
$$


Therefore for $k=1, \ldots, 4 n^{2}$ the last inequality gives

$$
\left\|x_{0}\right\|_{k}>1 / 2 n
$$

hence,

$$
1 \geq\left\|x_{0}\right\|=\left(\sum_{k=1}^{\infty}\left\|x_{0}\right\|_{k}^{2}\right)^{1 / 2} \geq\left(\sum_{k=1}^{4 n^{2}}\left\|x_{0}\right\|_{k}^{2}\right)^{1 / 2}>1 .
$$

This is a contradiction proving the Claim. The proof of the lemma follows from this claim and Theorem 1.7.

The next result is an immediate consequence of Theorem 1.8.

3.6. LemMA. For any U.E.C. subset $K$ of a B-space with an unconditional basis the set $\mathscr{E}_{1}(K)$ is also U.E.C.

We now pass to some consequences of Lemmas 3.5 and 3.6. Certain of them improve corresponding results from [6].

3.7. Corollary. Every U.W.C. operator T: $X \rightarrow Y$ can be factorized through a reflexive $B$-space $R(T)$ with an equivalent U.C.E.D. norm. If, furthermore, $Y$ has an unconditional basis, then $R(T)$ may be chosen with an unconditional basis.

3.8. Proposition. For any reflexive $B$-space $X$ with an unconditional basis $\left\{x_{\gamma}\right.$ : $\gamma \in \Gamma\}$ there exists a reflexive $B$-space $R(X)$ with an unconditional basis, an equivalent U.C.E.D. norm and a linear bounded one-to-one operator $T: R(X) \rightarrow X$ with dense range.

Proof. We set $K=\left\{e_{\gamma}: \gamma \in \Gamma\right\} \cup\{0\}$ which is a U.E.C. subset of $X$. Consider the factoring space $R(X)$ corresponding to the set $W=\mathscr{E}_{1}(\overline{\operatorname{co}}(K \cup(-K)))$. The space $R(X)$ has an unconditional basis, and from Lemmas 3.5, 3.6 and Theorem 2.3 we get that $R(X)$ admits an equivalent U.C.E.D. norm. Finally, it is clear that the identity map $j: R(X) \rightarrow X$ has dense range.

3.9. Corollary. A B-space $X$ is W.C.G. iff there exists a reflexive $B$-space $R(X)$ with an unconditional basis, a U.C.E.D. norm and a linear bounded one-to-one T: $R(X) \rightarrow X$ with dense range.

Proof. From the results of [6] there exists a space $\bar{R}(X)$ satisfying all the desired properties except that of the U.C.E.D. norm. Applying Proposition 3.8 to space $\bar{R}(X)$ we get the desired space $R(X)$.

3.10. REMARK. We conclude this section by giving two simple examples. The first corresponds to Corollary 3.7 and the second to Corollary 3.9. Both show that, for the spaces $R(T)$ and $R(X)$, it is not always possible to admit an equivalent uniformly convex norm.

3.11. Example. We set $X=\left(\sum_{q \in Q} \oplus l^{q}(\Gamma)\right)_{2}$ where $Q$ denotes the rational numbers $q$ so that $1<q<\infty$ and $\Gamma$ is an infinite set. It is clear that the unit ball of $X$ is a U.E.C. set and its norm is U.C.E.D.

The space $X$ does not admit an equivalent uniformly convex norm. Hence the identity map $j: X \rightarrow X$ is not factorized through any uniformly convexifiable $B$-space. 
3.12. Remark. Assume that the set $\Gamma$ in the previous example is uncountable. Then the conclusion is strengthened as follows:

The unit ball of the space $X$ is not affinely homeomorphic in the weak topology to a subset of any uniformly convex $B$-space.

We may derive this result by showing first that every uniformly convex $B$-space is mapped by a linear bounded one-to-one operator into $l^{p}(\Delta)$ for a suitable $1<p<\infty$ [12].

Therefore any affine homeomorphism of $S_{X}$ into a uniformly convex space $Y$ defines a bounded linear one-to-one operator $T: X \rightarrow l^{p}(\Delta)$ for a suitable $p$ with $1<p<\infty$. This contradicts the choice of the space $X$.

3.13. ExAmple. Consider the space $C(K)$ of continuous real valued functions on a compact set $K$ which is Eberlein compact and not U.E.C. Then the reflexive $B$-space $R(C(K))$ defined by Corollary 3.9 is not uniformly convexifiable.

To see this let $T: R(C(K)) \rightarrow C(K)$ be a linear bounded operator with dense range. We denote by $T^{*}: M(K) \rightarrow R^{*}(C(K))$ the conjugate of $T$ which is one-to-one and $w^{*}$-continuous. Hence $K$ is homeomorphic to a weakly compact subset of the space $R^{*}(C(K))$. Therefore $R^{*}(C(K))$ is not uniformly convexifiable and by known results $R(C(K))$ also is not uniformly convexifiable.

4. In this section we prove that the weakly compact subsets of $L^{1}(\mu)$-spaces are U.E.C.

We start with some preliminaries.

4.1. We denote by $L^{1}\{-1,1\}^{I}$ the $B$-space of all integrable real valued functions defined on the compact group $\{-1,1\}^{I}$ supporting the Haar measure. We denote by $\pi_{\{i\}}:\{1,1\}^{I} \rightarrow\{-1,1\}$ the projection onto the $i$-coordinate. For any $S \subset I$ finite set the $S$-Walsh function is denoted by $W_{S}=\Pi_{i \in S} \pi_{\{i\}}$. It is well known that the family $\left\{W_{S}: S \subset I, S\right.$ finite $\}$ defines a bounded one-to-one operator

$$
T: L^{1}\{-1,1\}^{\prime} \rightarrow c_{0}(\Gamma),
$$

where $\Gamma$ denotes the set of all finite subsets of $I$ and, for $S \in \Gamma, T f(S)=\int f \cdot W_{S} \cdot d \mu$.

4.2. We will use the following well-known characterization of weakly compact subsets of $L^{1}(\mu)$ :

A set $K \subset L^{1}(\mu)$ is weakly relatively compact iff for each $\varepsilon>0$ there exists $\delta(\varepsilon)>0$ so that for all $f \in K$ and measurable sets $V$ with $\mu(V)<\delta(\varepsilon)$ we have $\int_{V}|f| d \mu<\varepsilon$.

The first author thanks Professor S. Pichoredes for his help in the proof of the next lemma.

4.3. Lemma. Let $\mu$ be a probability measure and $f, f_{1}, f_{2}, \ldots, f_{n}$ elements of $L^{\infty}(\mu)$ so that

(1) $f=\sum_{i=1}^{n} a_{i} f_{i},\|f\|_{1} \leq 1,\left\|f_{i}\right\|_{\infty}=\left\|f_{i}\right\|_{2}=1$ and

$$
\int f_{i} f_{j} d \mu=0 \text { for } i \neq j
$$


(2) Given $\varepsilon>0$, there exists $k \in \mathbf{N}$ so that for all measurable sets $V$ with $\mu(V)<1 / k$

$$
\int_{V}|f| d \mu<\varepsilon
$$

Put $J=\left\{i<n:\left|a_{i}\right|>2 \varepsilon\right\}$; then $|J|<(k / \varepsilon)^{2}$.

Proof. We set $A=\{x:|f(x)|>k\}$. We notice that $k \mu(A) \leq \int_{A}|f(t)| d \mu \leq 1$, hence $\mu(A)<1 / k$. Assume, on the contrary, that the conclusion of the lemma fails.

Consider the function $\phi=f I_{A^{c}}$ and observe that

$$
\|f-\phi\|_{1}<\varepsilon .
$$

Extend the family $\left\{f_{i}\right\}_{i=1}^{n}$ to a complete biorthogonal system of $L^{2}(\mu)$, say $\left\{f_{i}\right\}_{i=1}^{n} \cup\left\{\bar{f}_{j}\right\}_{j \in J}$. Therefore

$$
\phi=\sum_{i=1}^{n} b_{i} f_{i}+\sum_{j \in J} b_{j} \bar{f}_{j} .
$$

By (*) it follows that for $1 \leq i \leq n$ we have $\left|b_{i}-a_{i}\right| \leq \varepsilon$, and therefore

$$
k \geq\|\phi\|_{\infty} \geq\|\phi\|_{2} \geq\left(\sum_{J} b_{i}^{2}\right)^{1 / 2} \geq \varepsilon|J|^{1 / 2} .
$$

This is a contradiction proving the lemma.

4.4. THEOREM. Every weakly compact subset of $L^{1}\{-1,1\}^{\prime}$ is U.E.C.

Proof. Let $K$ be a weakly compact subset of $L^{1}\{-1,1\}^{I}$ with $\|f\| \leq 1$ for all $f \in K$. We denote by $T: L^{1}\{-1,1\}^{I} \rightarrow c_{0}(\Gamma)$ the operator defined in 4.1. For fixed $\varepsilon>0$ there exists, by 4.2 , a $k \in \mathbf{N}$ so that for every $f \in K$ and any measurable set $V$ satisfying $\mu(V)<1 / k$ we have $\int_{V}|f| d \mu<\varepsilon / 4$. We claim that

$$
|\{S \in \Gamma: T f(S)>\varepsilon\}|<4 k^{2} / \varepsilon^{2} .
$$

To show this choose a finite linear combination $h=\sum_{i=1}^{n} a_{i} W_{S_{i}}$ of Walsh functions so that $\|h-f\|<\varepsilon / 4$ and $\|h\| \leq 1$. Notice that $\int_{V}|h| d \mu<\varepsilon / 2$ whenever $\mu(V)<1 / k$; therefore, applying Lemma 4.3 we get

$$
\left|\left\{i: 1 \leq i \leq n\left|a_{i}\right|>\varepsilon\right\}\right|<4 k^{2} / \varepsilon^{2} .
$$

We also remark that

$$
T h=\sum_{i=1}^{n} a_{i} e_{S_{i}}
$$

(Here $e_{S_{1}}$ denotes the $S_{i}$-unit vector of $c_{0}(\Gamma)$.)

Let $\left\{h_{n}\right\}_{n=1}^{\infty}$ be a sequence of linear combinations of Walsh functions so that $h_{n} \stackrel{\|\cdot\|_{1}}{\rightarrow} f$ and $\left\|f-h_{n}\right\|_{1}<\varepsilon / 4$. Then $T h_{n} \rightarrow T f$ and each $T h_{n}$ satisfies property (*). Hence $T f$ itself satisfies property (*), and by Theorem 1.7 we get the desired result. The proof of the theorem is now complete. 
4.5. REMARK. (a) A combination of Maharam's theorem and Kakutani's representation theorem allows us to extend the result of Theorem 4.4 to arbitrary $L^{1}(\mu)$-space.

(b) Notice that the proof of Theorem 4.4 actually shows that if $K \subset L^{1}\{-1,1\}^{I}$ is a weakly compact set and $\varepsilon>0$ is given, then there exists a natural number $N(K, \varepsilon)$ so that for all $f \in K$

$$
|\{S \subset I:|T f(S)|>\varepsilon\}|<N(K, \varepsilon)
$$

Here $T$ denotes the operator defined in 4.1.

We finish this section with some remarks related to possible renormings of $L^{1}(\mu)$.

4.6. Remark. (a) As we have noticed in Remark 2.8 we do not know whether or not every $L^{1}(\mu)$-space admits an equivalent U.C.E.D. norm. We can show that Day's strictly convex norm [8] on $L^{1}(\mu)$ fails to be U.C.E.D.

(b) It can be shown that Day's norm on $L^{1}(\mu)$ satisfies the fixed point property. More precisely every convex weakly compact subset of $L^{1}(\mu)$ has normal structure [8]. This is proved by using Theorem 4.4 and [19, Proposition 3.4].

\section{REFERENCES}

1. A. Abramovich, Weakly compact sets in topological $K$-spaces, Theor. Funktsii Funktsional. Anal. I Prilozhen 15 (1972), 27-35.

2. D. Amir and J. Lindenstrauss, The structure of weakly compact sets in Banach spaces, Ann. of Math. (2) 88 (1968), 35-46.

3. S. Argyros, On nonseparable Banach spaces, Trans. Amer. Math. Soc. 210 (1982), 193-216.

4. Y. Benyamini and T. Starbird, Embedding weakly compact sets into Hilbert space, Israel J. Math. 23 (1976), 137-141.

5. Y. Benyamini, M. E. Rudin and M. Wage, Continuous image of weakly compact subsets of Banach spaces, Pacific J. Math. 70 (1977), 309-324.

6. W. J. Davies, T. Figiel, W. B. Johnson and A. Pelczynski, Factoring weakly compact operators, J. Funct. Anal. 17 (1974), 311-327.

7. M. Day, R. C. James and S. Swaminathan, Normed linear spaces that are uniformly convex in every direction, Canad. J. Math. 23 (1971), 1051-1059.

8. J. Diestel, Geometry of Banach spaces, Selected Topics, Lecture Notes in Math., vol. 485. Springer-Verlag, Berlin and New York.

9. W. F. Eberlein, Weak compactness in Banach spaces. I, Proc. Nat. Acad. Sci. U.S.A. 33 (1947), $51-53$.

10. T. Figiel, W. Johnson and L. Tzafriri, On B-lattices and spaces having local unconditional structure with application to Lorentz functions spaces, J. Approx. Theory 13 (1975), 395-412.

11. A. L. Garkavi, On the Chebyshev center of a set in a normed space, Investigations on Cortemporary Problems in the Constructive Theory of Function, Moscow, 1981, pp. 328-331.

12. R. C. James, Super reflexive spaces with hases, Pacific J. Math. 41 (1972), 409-419.

13. D. N. Kutzarova and S. L. Troyanski, Reflexive Banach spaces without equivalent norms which are uniformly convex or uniformly differential in every direction. Studia Math. 72 (1982), 92-95.

14. J. Lindenstrauss and L. Tzafriri, Classical Banach spaces, Lecture Notes in Math., vol. 338 , Springer-Verlag, Berlin and New York.

15. R. Phelps, Lectures in Choquet's theorem, Van Nostrand Math. Studies, Vol. 7, Van Nostrand, Princeton, N. J.

16. H. P. Rosenthal, The hereditary problem for weakly compactly generated Banach spaces, Compositio Math. 28 (1974), 83-111.

17. N. Shanin, On the product of topological space, Trudy Mat. Inst. Akad. Nauk. SSSR 24 (1948), 112 pages (Russian). 
18. S. L. Troyanski, On uniform convexity and smoothness in every direction in non-separable Banach spaces with an unconditional basis, C. R. Acad. Bulgare Sci. 30 (1977), 1243-1246.

19. On locally uniformly convex and differentiable norms in certain non-separable Banach spaces, Studia Math. 37 (1971), 173-180.

20. V. Zizler, On some rotundity and smoothness properties of Banach spaces, Dissertationes Math. 87 (1971), 5-33.

Department of Mathematics, University of Crete, Iraklion, Crete, Greece

Mathematical Institute, Athens University, Solonos 57, Athens 27, Greece 